\title{
EVALUATING COMPUTATIONAL COSTS WHILE HANDLING DATA AND CONTROL PARALLELISM
}

\author{
SONIA CAMPA \\ Dept. Computer Science, University of Pisa, Largo B.Pontecorvo 3 \\ Pisa, 56123, Italy
}

\begin{abstract}
The aim of this work is to introduce a computational costs system associated to a semantic framework for orthogonal data and control parallelism handling. In such a framework a parallel application is described by a semantic expression involving in an orthogonal manner both data access and control parallelism abstractions. The evaluation of such an expression is driven by a set of rewriting rules each of which is combined with a computational cost. We present how to proceed in the evaluation of the final cost of the application as well as how such information together with the semantic framework capabilities can be exploited to increase the overall performance.
\end{abstract}

\section{Introduction}

In the latest years, the literature has widely recognized all the advantages provided by the structured parallel programming paradigm [1] in which an application is given as a composition of (a set of) recurrent patterns whose control behavior is well known and predictable, while the functional behavior depends on the functional parameters provided by the user who is instantiating the patterns. Stating in advance the control behavior of an application allows, in principle, to statically make some kind of prediction on its computational costs and thus, to intervene on the application graph in order to increase the overall performance (for example, by augmenting the parallelism degree). On the other hand, a parallel application have also to deal with data access concerns that can heavily influence both the programming phase and the final computational costs. Unfortunately, the most works regarding structured parallel programming environments, define a two-tier architecture model in which data accesses have to be heavily detailed on top of control abstractions $[2,3]$ or vice versa $[4,5,6,7]$. As a consequence, the model of parallelism is a mixture of strictly coupled data and control concerns difficult to formalize and, hence, to statically and/or dynamically analyze for optimization purpose.

In [8] we have sketched a programming model in which parallel applications are built by keeping orthogonal data and task parallel concerns through a set of 
abstract mechanisms. In [9] we have given a preliminary formalization of the model, pointing out that the evaluation of a parallel application is described by a sequence of transformations (i.e. inference rules) on the application graph involving abstract mechanisms for both expressing control concerns and data access concerns. The contribution given by this work is a more clear specification of the semantics and the introduction of a computational costs system provided by the semantics. In fact, the former formalizes both mechanisms for describing control concerns and data access concerns, thus reaching three goals:

- giving a formal description of the abstract mechanisms that handle data and task parallel concerns leads to a clear, unambiguous description of the programming model, regardless any further implementation choice

- the semantics can assign some kind of evaluation costs about the use of our abstract mechanisms use in order to statically estimate the computational cost of the whole application.

- the semantics is enriched with a set of rewriting rules about graph structures that assess if two graphs are functionally equivalent and each graph has associated an estimation of its computational cost. Rewriting rules and static estimations can drive static and dynamic improvements of the overall performance. For example, it can be proved that a certain control structure is equivalent and more efficient than the one declared by the user, thus driving a transparent rewriting process of the graph application at running or compile time.

This work is structured as follows: Section gives an overall introduction to the programming model we are assessing. Section presents the basic of both our semantics and our costs system, in particular which operators are involved as well as how they can be used to describe and computationally evaluate parallel applications. Section shows a simple case study as well as how our cost system can lead to the estimation of the final computational costs. Section concludes the presentation by giving an overview of related and future work.

\section{The programming model}

Our programming model is fully described by the tuple $\mathcal{M}=\langle\mathcal{A}, \mathcal{V}, \mathcal{I}, \mathcal{C}\rangle$ where $\mathcal{A}$ stands for a set of abstract data types; $\mathcal{V}$ stands for the set of views on abstract data types; $\mathcal{I}$ stands for a set of iterators for accessing views; $\mathcal{C}$ stands for the set of collectives (or control primitives) which allow to describe structured functional graphs (i.e. graphs built composing the available collectives).

The first three mechanisms fully describe the data parallel concerns of the application. An instance of a given abstract data type is a representation of the raw input data abstracting from their actual implementation and/or distribution. Different kind of typed views can be declared on top of such instance, thus providing a logical organization of the raw input data and a set of operators to manipulate them. Since the view is independent from the actual implementation of data, such separation gives many hooks to optimize the implementation of both the view's 
operators and the data itself.

Each view provides a set of typed iterators on its items. An iterator is an object exposing a set of operators to get items from the view, coherently with the view type and with a given pattern of access. Moreover, each type of iterator can be specialized in order to get the items in a whole or sequentially. For example, an array view provides iterators to get singletons or block of items, while a tree view provides iterators to get subtrees, children or siblings of a given node and so on. Such items can be returned by the iterator one-by-one or as a whole, depending on the specialized behavior of the iterator.

The task parallel behavior of the application is described by using the set of primitives $\mathcal{C}$, a collection of composable patterns of control plus sequential functions types. The basic idea is that the user application can be formalized as a graph in which the nodes are sequential or parallel modules and the arcs are functional (i.e. data) dependencies among modules. Such an application graph is constructed by selecting and composing the patterns provided by the set of primitives $\mathcal{C}$. Pipe (pipelines of stages), Apply-to-all (application of a function to all the elements of an input data set in parallel), Comp (sequential composition), Reduce (function reduction) etc. populate $\mathcal{C}$.

For example, let us suppose we have an application that applies the function $S_{2}\left(S_{1}(p x)\right)$ to each pixel $p x$ of the images belonging to an input stream of images. Such application can be written as a pipeline of two stages: the first stage applies $S_{1}(p x)$ to all the pixels of the current image; the second stage applies $S_{2}$ to all the pixels updated by the first one. Hence, the global application results in a Pipe of two stages, each of which is an Apply-to-all computing a given sequential function.

Summarizing, our programming model allows to exploit data parallelism by selecting appropriate abstract data types, views and iterators and without keeping in mind how to evaluate the functions that will use them. On the other hand, the application can be completely described by composing its computational graph regardless details about accesses and/or distribution of input data.

Nevertheless, since each module in the graph accesses only the piece of data that flows from its parent, its input can be easily provided by an iterator handling such portion, thus coupling in a very high level manner control and access behavior. In fact, in our programming model the harmonization between data and task parallelism is given by coupling each selected primitive with one or more iterators. The internal behavior of each primitive depends on the specific pair control patterniterator by which it is composed. For example, let us consider an application that can be evaluated applying in parallel the same function to all the items belonging to an array view. As mentioned before, such simple pattern is represented in $\mathcal{C}$ by the Apply-to-all primitive. However, more pragmatically, the application can be written instantiating a set of processing elements each of which applies the function to an independent task of the input data set, thus exploiting a plain task parallel behavior. Otherwise, the same application can be written as a plain data parallel computation, thus applying the function to all the items of the data set in parallel. The former specialized pattern is called farm in the skeleton community while the latter is called map. In our model, a farm computation can be described by 
combining the Apply-to-all primitive with a sequential iterator (thus, applying a function to all the elements accessed one-by-one). Instead, a map computation can be obtained by coupling the same primitive with a parallel iterator: in this case all the elements are accessed as a whole and the function is applied in parallel on each of them.

\section{Semantics}

The formal semantics of our programming model is given by populating the tuple $\langle\mathcal{A}, \mathcal{V}, \mathcal{I}, \mathcal{C}\rangle$. Each type of the tuple is represented as a set plus some operators to manipulate its elements.

Since $\mathcal{A}$ is the type representing the raw input data, it can be modeled as a set of objects of a generic type $\mathcal{O}$ provided with canonical operators for creating a set, querying its content, inserting, deleting, updating elements, and so on. Tab.1 summarize the formalization of type $\mathcal{A}$. The view abstraction is provided by the

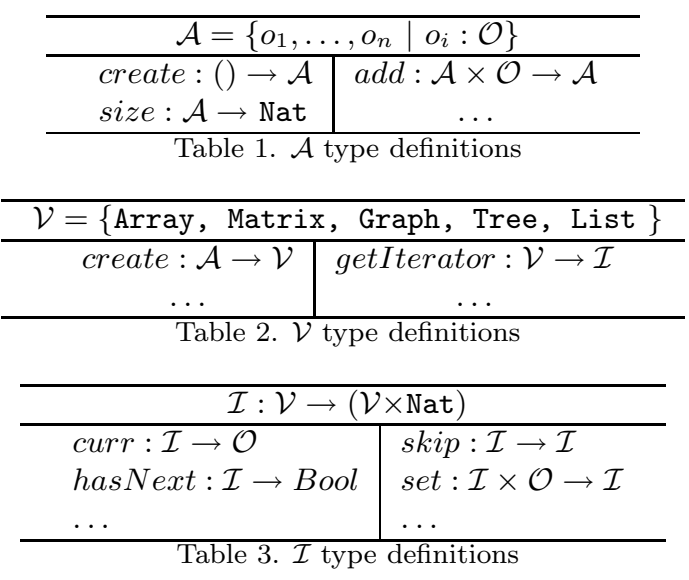

set $\mathcal{V}$ of view types that map a given $\mathcal{A}$ object to a $\mathcal{V}$ object. At the moment, the provided view types are Array, Matrix, Graph, Tree and List. For example, given $s=\left\{s_{1}, \ldots, s_{\text {size }(s)}\right\} \in \mathcal{A}$, and given $g \in$ Nat as a grain value divisor of size $(s)$, the Array $(s, g)$ type is constructed by the mapping function

$$
\mathcal{A} \times \mathcal{N} \rightarrow \operatorname{Array}(\mathrm{s}, \mathrm{g})=<<s_{1}, \ldots, s_{g}>, \ldots,<s_{\text {size }(s)-g}, \ldots, s_{\text {size }(s)}>>
$$

Each view $v \in \mathcal{V}$ is a factory for a set of iterators on its structure, thus it provides kind of getIterator operators (see Tab.2). Generally speaking, an iterator is a pair $(v, p)$ where $v \subseteq \mathcal{V}$ is the (sub)view on which it is defined and $p \in$ Nat is a pointer to the next accessible item of its range.

Tab. 3 shows some operations to handle an iterator object. The curr operator returns the current item pointed by $p$. If the iterator has a parallel behavior, $p$ "points" in parallel to all the items so that they are taken as a whole; otherwise $p$ points also to the current item and the operator returns it as a singleton.

The set of collectives $\mathcal{C}$, as mentioned before, includes well known patterns of control as for example Pipe, Apply-to-all, Comp etc. Generally speaking, each 
collective can be viewed as a function

$$
\mathcal{C}: \mathcal{I} \times(\mathcal{C} \cup \text { Function }) \rightarrow \mathcal{V}
$$

that gets a sequential function (let us call Function its type) or a nested control primitive and a set of values (i.e. the ones given by one (or more) iterator(s)) and returns the view obtained by applying the second argument to the set of values. As explained in Section, the parallel access behavior by which such values will be accessed is encapsulated by the input iterator instance.

In our semantic model, collectives, iterators and related operators are defined in terms of semantics expressions whose evaluation is given by means of inference rules stating functional equivalences between expressions. $T_{1}$ is a general example of a rule of ours:

$$
T_{1}: \frac{C\left(E_{1}\right)}{E_{1} \rightarrow_{c} E_{2}}
$$

The expression $E_{1}$ is transformed by rule $T_{1}$ into the equivalent expression $E_{2}$, if some condition $C$ on $E_{1}$ holds. Each transformation is provided with a cost $c$ and the semantics supplies a cost function $\mathcal{E}: \mathcal{C} \rightarrow$ Nat that associates a costs to each primitive $\mathcal{C}$ in the following way. Let $\mathcal{C} \rightarrow_{c_{1}} E_{1} \rightarrow_{c_{2}} \ldots \rightarrow_{c_{k}} v$ be the chain of transformations for evaluating $\mathcal{C}$ up to the final result $v$. Then,

$$
\mathcal{E}(\mathcal{C})=\sum_{i=1}^{k} c_{i}
$$

that is, the computational cost for evaluating $\mathcal{C}$ is the sum of the costs of all the partial transformations applied to transform $\mathcal{C}$ into $v$.

Let us give some example of how this relation works and on which operators. As mentioned before, the iterator is represented as a pair $(\bar{x}, p)$ where $\bar{x}=<$ $x_{1}, \ldots, x_{n}>$. The curr operator is in charge of returning (thus is functionally equivalent to) the current element of its range. If the iterator it is an instance of SeqIterator, then curr will only return the $p$-th element of the range; if the it is of type ParIterator, then the whole range is returned. The curr operator is evaluated by the following rules:

$$
\frac{i t=\left(<x_{1}, \ldots, x_{n}>, p\right): \text { SeqIterator }}{\operatorname{curr}(i t) \rightarrow c_{c u r r} x_{p}} \quad \frac{i t=(x, p): \text { ParIterator }}{\operatorname{curr}(i t) \rightarrow c_{c u r r} x}
$$

In the formulae given above $c_{\text {curr }}$ represent the cost for evaluating the curr operator. Thus, quantitatively speaking, such cost includes costs for retrieving and accessing data.

Another operator that will often occur in the following is skip. Its role is simply to skip the pointer to the next position in the iterator state (or returns $\perp$ if any), without returning values.

$$
\frac{i t=(x, p) \wedge i t^{\prime}=(x, p+1)}{\operatorname{skip}(i t) \rightarrow c_{s k i p} i t^{\prime}} \quad \frac{i t=(x, \operatorname{size}(x))}{\operatorname{skip}(i t) \rightarrow c_{s k i p} \perp}
$$

In this case $c_{\text {skip }}$ represents just the cost for updating the internal state of $i t$. 
The operator that describes the parallel evaluation of two functions $f$ and $g$ is given by $f \| g$ (par operator). For example, two of the rules leading the transformation of such an operator are the ones assessing the evaluation order:

$$
a . \frac{g(x) \rightarrow_{c_{1}} y^{\prime} \wedge f(x) \rightarrow_{c_{2}} x^{\prime} \wedge c=\max \left\{c_{1}, c_{2}\right\}}{h(g(x))\left\|h^{\prime}(f(x)) \rightarrow_{c} h\left(y^{\prime}\right)\right\| h^{\prime}\left(x^{\prime}\right)} \quad b \cdot \frac{g(y) \rightarrow_{c_{1}} y^{\prime}}{x^{\prime} \| g(y) \rightarrow_{c_{1}}<x, y^{\prime}>}
$$

The evaluation of par is strict i.e. before evaluating it, all its arguments have to be completely evaluated (rule 4-a). Moreover, the evaluation of par produces a new view by appending all the partial results in a new data set (rule 4-b).

The semantics includes the operator chain $(\oplus)$ that, given a set of functions $f_{1}, \ldots, f_{m}$ represented by the sequential iterator $i t_{f}=\left(\left\langle f_{1}, \ldots, f_{m}\right\rangle, p\right)$ and an object $x \in \mathcal{O}$, evaluates $f_{m}\left(. .\left(f_{1}(x)\right)\right.$..). Such operator is transformed as follows:

$$
\frac{\operatorname{hasNext}\left(i t_{f}\right) \rightarrow_{c_{h}} \text { true }}{i t_{f} \oplus x \rightarrow c_{\oplus} \operatorname{skip}\left(i t_{f}\right) \oplus \operatorname{curr}\left(i t_{f}\right)(x)} \quad \frac{\operatorname{hasNext}\left(i t_{f}\right) \rightarrow_{c_{h}} \text { false }}{i t_{f}^{(p)} \oplus x \rightarrow_{c_{\oplus}} x}
$$

The chain operator is called recursively on the updated state of $i t_{f}$ and on the last evaluated object, till there are functions in $i t_{f}$ to apply.

The composition of par and chain allows to define the Pipe collective, e.g. a pattern of control that applies the composition of the functions represented by the iterator $i t_{f}$, to the stream of input values represented by the sequential iterator $i t_{d}$.

$$
\begin{aligned}
& \frac{\operatorname{hasNext}\left(i t_{d}\right) \rightarrow c_{h} \text { true } \wedge c_{h}=c_{\text {pipe }}}{\operatorname{Pipe}\left(i t_{f}, i t_{d}\right) \rightarrow c_{\text {pipe }} i t_{f} \oplus \operatorname{curr}\left(i t_{d}\right) \| \operatorname{Pipe}\left(i t_{f}, \operatorname{skip}\left(i t_{d}\right)\right)} \\
& \frac{\text { hasNext }\left(i t_{d}\right) \rightarrow{ }_{c_{h}} \text { false } \wedge c_{h}=c_{\text {pipe }}}{\operatorname{Pipe}\left(i t_{f}, i t_{d}\right) \rightarrow c_{p i p e}<>}
\end{aligned}
$$

As it can be seen, for each transformation call of Pipe, the evaluation proceeds in two parallel steps * the chain operator is applied to the functions provided by $i t_{f}$ and the value currently pointed by $i t_{d}$ through the curr operator. In the meanwhile, a recursive call of the operator Pipe is evaluated together with the updated state of $i t_{d}$ (rule 6$)$. If there are no more elements to evaluate pointed by $i t_{d}$, then an empty view is returned (rule 7).

\section{Computational costs analysis}

Just to show the effectiveness of our rules, we will give a simple evaluation example. Let $i t_{f}=\left(<f_{1}, f_{2}>, 1\right), i t_{d}=\left(<x_{1}, x_{2}, x_{3}>, 1\right)$ and $i t^{(p)}$ be the notation for iterators stressing that the current state of it points to the $p$-th element. Tab.4 shows the evaluation process of Pipe $\left(i t_{f}, i t_{d}\right)$ labelling each transformation with its cost load.

The example demonstrates that evaluating a collective through our rules, not only drives through a formal description of the transformational process from one expression to a functionally equivalent one; it also allows to model how the computation evolves in time and to define the final computational cost. In fact, in a pipeline

\footnotetext{
${ }^{*}$ Since the evaluation of $\|$ is strict, all its arguments have to be evaluated, first
} 


\begin{tabular}{|c|c|c|c|}
\hline 1 & ${\operatorname{Pipe}\left(i t_{f}, i t_{d}\right)}$ & $\rightarrow$ cpipe & $i t_{f}^{(1)} \oplus \operatorname{curr}^{(1)}\left(i t_{d}\right) \| P i p e\left(i t_{f}\right.$, skip $\left.^{(2)}\left(i t_{d}\right)\right)$ \\
\hline 2 & & $\rightarrow \max \left\{c_{c u r r}, c_{\text {skip }}\right\}$ & $i t_{f}^{(1)} \oplus x_{1} \| \operatorname{Pipe}\left(i t_{f}, i t_{d}^{(2)}\right)$ \\
\hline 3 & & $\rightarrow \max \left\{c_{\oplus}, c_{\text {pipe }}\right\}$ & $\begin{array}{l}\operatorname{skip}^{(2)}\left(i t_{f}\right) \oplus\left(\operatorname{curr}^{(1)} i t_{f}\right)\left(x_{1}\right) \|\left(i t_{c} \oplus \operatorname{curr}^{(2)}\left(i t_{d}\right) \|\right. \\
\left.\quad \| \operatorname{Pipe}\left(i t_{f}, \operatorname{skip}^{(3)}\left(i t_{d}\right)\right)\right)\end{array}$ \\
\hline 4 & & $\rightarrow \max \left\{c_{\text {curr }}, c_{\text {skip }}\right\}$ & $i t_{f}^{(2)} \oplus f_{1}\left(x_{1}\right)\left\|\left(i t_{c} \oplus x_{2}\right)\right\| P i p e\left(i t_{f}, i t_{d}^{(3)}\right)$ \\
\hline 5 & & $\rightarrow \mathbf{c}_{\mathbf{f}_{1}}$ & $i t_{f}^{(2)} \oplus y_{1}\left\|\left(i t_{c} \oplus x_{2}\right)\right\| P i p e\left(i t_{f}, i t_{d}^{(3)}\right)$ \\
\hline 6 & & $\rightarrow \max \left\{c_{\oplus}, c_{\text {pipe }}\right\}$ & $\begin{array}{l}\operatorname{skip}^{(3)}\left(i t_{f}\right) \oplus \operatorname{curr}^{(2)}\left(y_{1}\right)\left\|\operatorname{skip}^{(2)}\left(i t_{f}\right) \oplus \operatorname{curr}^{(1)}\left(i t_{f}\right)\left(x_{2}\right)\right\| \\
\left\|i t_{f} \oplus \operatorname{curr}^{(3)}\left({ }^{(i t} d\right)\right\| \text { Pipe }\left(i t_{f}, \text { skip }^{(4)}\left(i t_{d}\right)\right)\end{array}$ \\
\hline 7 & & $\rightarrow \max \left\{c_{c u r r}, c_{\text {skip }}\right\}$ & $i t_{f}^{(3)} \oplus f_{2}\left(y_{1}\right)\left\|i t_{f}^{(2)} \oplus f_{1}\left(x_{2}\right)\right\| i t_{f} \oplus x_{3} \| P i p e\left(i t_{f}, i t_{d}^{(4)}\right)$ \\
\hline 8 & & $\rightarrow \max \left\{\mathbf{c}_{\mathbf{f}_{\mathbf{1}}}, \mathbf{c}_{\mathbf{f}_{\mathbf{2}}}\right\}$ & $i t_{f}^{(3)} \oplus y_{1}^{\prime}\left\|i t_{f}^{(2)} \oplus y_{2}\right\| i t_{f} \oplus x_{3} \| P i p e\left(i t_{f}, i t_{d}^{(4)}\right)$ \\
\hline 9 & & $\rightarrow \max \left\{c_{\oplus}, c_{p i p e}\right\}$ & $y_{1}^{\prime}\left\|s k i p^{(3)}\left(i t_{f}\right) \oplus \operatorname{curr}(2)\left(y_{2}\right)\right\| s k i p^{(2)}\left(i t_{f}\right) \oplus \operatorname{curr}(1){ }_{i t} f\left(x_{3}\right) \|[]$ \\
\hline 10 & & $\rightarrow_{\max \left\{c_{c u r r}, c_{\text {skip }}\right\}}$ & $y_{1}^{\prime}\left\|i t_{f}^{(3)} \oplus f_{2}\left(y_{2}\right)\right\| i t_{f}^{(2)} \oplus f_{1}\left(x_{3}\right) \|[]$ \\
\hline 11 & & $\rightarrow \max \left\{\mathbf{c}_{\mathbf{f}_{\mathbf{1}}}, \mathbf{c}_{\mathbf{f}_{\mathbf{2}}}\right\}$ & $y_{1}^{\prime}\left\|i t_{f}^{(3)} \oplus y_{2}^{\prime}\right\| i t_{f}^{(2)} \oplus y_{3} \|[]$ \\
\hline 12 & & $\rightarrow c_{\oplus}$ & $y_{1}^{\prime}\left\|y_{2}^{\prime}\right\| \operatorname{skip}^{(3)}\left(i t_{f}\right) \oplus \operatorname{curr}(2)\left(y_{3}\right)$ \\
\hline 13 & & $\rightarrow_{\max \left\{c_{\text {curr }}, c_{\text {skip }}\right\}}$ & $y_{1}^{\prime}\left\|y_{2}^{\prime}\right\| i t_{f}^{(3)} \oplus f_{2}\left(y_{3}\right)$ \\
\hline 14 & & $\rightarrow \mathrm{cf}_{2}$ & $y_{1}^{\prime}\left\|y_{2}^{\prime}\right\| i t_{f}^{(3)} \oplus y_{3}^{\prime}$ \\
\hline 15 & & $\rightarrow c_{\oplus}$ & $<y_{1}^{\prime}, y_{2}^{\prime}, y_{3}^{\prime}>$ \\
\hline
\end{tabular}

Table 4. Evaluation process of Pipe $\left(i t_{f}, i t_{d}\right)$

computation, the first step consists in evaluating $f_{1}\left(x_{1}\right)$; the second step consists in evaluating in parallel $f_{2}\left(f_{1}\left(x_{1}\right)\right)$ and $f_{1}\left(x_{2}\right)$ and so on. Once the full regimen has been reached, all the stages evaluate in parallel. This behavior is perfectly described in our semantics. In fact, in Tab.4 we have traced in bold font, the transformational steps describing the parallelism among stages: after some preliminary evaluations (rows 1-4), the first stage is evaluated, costing $c_{f_{1}}$ (row 5). Transformations 6-7 lead to the second and the third time-step of the pipeline (rows 8-11), in which $f_{1}$ and $f_{2}$ are evaluated in parallel. Moreover, the cost of these two steps is the maximum between the cost $c_{f_{1}}$ of evaluating $f_{1}$ and the $\operatorname{cost} c_{f_{2}}$ of evaluating $f_{2}$.

The final computational cost of the Pipe operator is given by adding all the intermediate transformational costs, so that

$$
\mathcal{E}\left(\text { Pipe }\left(i t_{f}, i t_{d}\right)\right)=6 c_{h}+5 c_{\text {curr }}+c_{f_{1}}+2 \max \left\{c_{f_{1}}, c_{f_{2}}\right\}+c_{f_{2}}
$$

Thus, the generalized formula

$$
\begin{aligned}
\mathcal{E}\left(\text { Pipe }\left(i t_{f}, i t_{d}\right)\right)= & (m+n+1) c_{h}+(m+n) c_{c u r r}+ \\
& \sum_{t=1}^{m-1} \max _{i \in[1, t]}\left\{c_{f_{i}}\right\}+ \\
& \sum_{t=m}^{n} \max _{i \in[1, m-1]}\left\{c_{f_{i}}\right\}+ \\
& \sum_{t=n+1}^{n+m-1} \max _{i \in[t-n+1, m]}\left\{c_{f_{i}}\right\}
\end{aligned}
$$

is the one evaluating the computational cost of a pipeline given an input stream of size $n \geq 1$ represented by the iterator $\left.i t_{d}=\left(<x_{1}, \ldots, x_{n}\right\rangle, 1\right)$ and $m \geq 1$ stages, globally represented by the iterator $i t_{f}=\left(<f_{1}, \ldots, f_{m}>, 1\right)$. The inner sums measure at each time step $i \in[1, m+n-1]$, the maximum paid cost and they add it to the cost accumulated in the preceding steps. 
It has to be pointed out that in the previous example we have shown how our semantics allow to derive a computational cost as soon as a primitive has been defined. Each time such primitive recurs as part of a more complex graph or as a graph by its own, the formula statically estimated by our semantics framework can be taken into account to automatically estimate its computational cost of the (sub)graph.

Provided that the cost evaluation of each collective in $\mathcal{C}$ can be easily estimated by the semantics, we are able to quantify the computational costs of two functionally equivalent graphs and to choose the more efficient one for our execution. In fact, we can prove, for example that $\alpha\left(P i p e\left(i t_{f}, i t_{\text {seq }}^{\prime}\right), i t_{\text {par }}\right) \equiv$ Pipe $\left(i t_{\alpha}, i t_{\text {seq }}\right)$ (a Map of Pipe is functionally equivalent to a Pipe of Map) but also that $\mathcal{E}\left(\alpha\left(\right.\right.$ Pipe $\left.\left.\left(i t_{f}, i t_{\text {seq }}^{\prime}\right), i t_{\text {par }}\right)\right)<\mathcal{E}\left(\operatorname{Pipe}\left(i t_{\alpha}, i t_{\text {seq }}\right)\right)$. Hence, each time a user instantiates a pipelines of maps, the rewriting rule can be adopt to improve the performance. In other cases, as for example $\alpha\left(\operatorname{Pipe}\left(i t_{f}, i t_{s e q}^{\prime}\right), i t_{p a r}\right) \equiv \alpha\left(\left(f_{1} ; \ldots ; f_{m}\right), i t_{p}\right)$ (equivalence between a Map of Pipe versus a Map of the same stages composed sequentially), we know that the first Map costs more with respect to the second one: in fact, they exploit $O(m+n)$ and $O(m)$, respectively. Thus the actual convenience of instantiating one graph respect to the other depends on the input size.

\section{Related work and conclusions}

We have presented a semantic framework for the description of a programming model in which data access and control patterns (the ones composing the application graph) are described through independent abstract mechanisms. The model exposes a set of semantics expressions that define our mechanisms and a set transformations stating how a given expression evolves at running time. Such transformation, given as an inference rule, allows to statically define a functional equivalence between the left and the right member of the transformation. Moreover, the semantics provides a cost model assigning a cost to each transformation. As a consequence, such an inference rules system provides the basis for the definition of an evaluation function for assigning a cost to each pattern of control involved in our application, thus to the whole application itself.

Hence, our framework is able to statically (or even dynamically) evaluate the user application graph, to estimate the costs of its execution and, if it is the case, to apply smart rewriting rules to transform the user graph in a functionally equivalent graph but exposing a better performance.

The idea of formally evaluating in some way the computational cost of an application graph is not new, especially in the field of structured parallel programming. Several works has already presented in the past aiming at defining in some sense the behavior of a parallel structure $[10,11]$ and, at possibly associating a cost value to its execution [12]. The novelty introduced with our approach is given by a semantic model able to both reproduce the evolution of an application graph execution and, in the same time, to estimate and/or rewrite such evolution in an easy and comprehensible way.

We are planning to provide our programming model with a suitable set of well known transformations that can be eventually applied to the user defined application in a transparent manner. In fact, the final goal is to have a programming framework in 
which performance optimizations are automatically done on the basis of the static (and dynamic) analysis of the user application graph. On the semantic side, we are working on enriching the framework with new primitives and abstract data types as well as in describing more complex case studies evidencing the power of our approach.

\section{References}

[1] Murray Cole. Algorithmic Skeletons: structured management of parallel computation. Monograms. Pitman/MIT Press, Cambridge, MA, 1989.

[2] S. Bromling, S. MacDonald, J. Anvik, J. Schaeffer, D. Szafron, and K. Tan. Patternbased parallel programming, August 2002. 2002 International Conference on Parallel Programming (ICPP-02), Vancouver, British Columbia, August 2002.

[3] Manuel Díaz, Bartolomé Rubio, Enrique Soler, and José M. Troya. Integrating task and data parallelism by means of coordination patterns. Int. Conf. on Parallel Programming (ICPP-02), LNCS 2026:16, 2001.

[4] Ian Foster, David R. Kohr, Jr., Rakesh Krishnaiyer, and Alok Choudhary. A librarybased approach to task parallelism in a data-parallel language. Journal of Parallel and Distributed Computing, 45(2):148-158, 15 September 1997.

[5] H. Kuchen. A skeleton library. In Proc. of Euro-Par 2002, volume 2400 of LNCS, pages 620-628, 2002. Paderborn, Germany.

[6] H. Kuchen and M. Cole. The integration of task and data parallel skeletons. Parallel Processing Letters, 12(2):141, June 2002.

[7] L. Rauchwerger, F. Arzu, and K. Ouchi. Standard templates adaptive parallel library (STAPL). 4th International Workshop on Languages, Compilers, and Run-Time Systems for Scalable Computers, LNCS 1511, 1998.

[8] S. Campa and M. Danelutto. A framework for orthogonal data and control parallelism exploitation. In Proceedings of ICCSA 2004, Springer Verlag, LNCS, Vol. 3046, pages 1295-1300, August 2004.

[9] Sonia Campa. A formal framework for orthogonal data and control parallelism handling. In Int. Conf. on Computational Science (ICCS 2005), eds. V. Sunderman, D. van Albada, P. Sloot, and J. Dongarra, LNCS, Springer, to appear

[10] C. Lengauer, M. Aldinucci, and S. Gorlatch. Towards parallel programming by transformation: The FAN skeleton framework. In Parallel Algorithms and Applications, 16(2-3):87-122, Gordon and Breach (Taylor and Francis group), March 2001. ISSN 1063-7192.

[11] S. Gilmore A. Benoit, M. Cole and J. Hillston. Evaluating the performance of skeletonbased high level parallel programs. In Proceedings of the Intl. Conference on Computational Science (ICCS 2004), volume 3038 of LNCS, pages 289-296. Springer Verlag, 2004.

[12] M. Aldinucci and M. Danelutto. Rewriting skeleton programs: How to evaluate the data-parallel stream-parallel tradeoff. In Proc. of the 1st Intl. Workshop on Constructive Methods for Parallel Programming, MIP 9805 pages 44-58, June 1998. 